PUPT-1947

DAMTP-2000-76

\title{
Interacting Black Holes
}

\author{
Miguel S. Costa円 \\ Joseph Henry Laboratories \\ Princeton University \\ Princeton, New Jersey 08544, USA \\ Malcolm J. Perry \\ D.A.M.T.P., Center for Mathematical Sciences \\ University of Cambridge \\ Wilberforce Road, Cambridge CB3 OWA, UK
}

\begin{abstract}
We revisit the geometry representing $l$ collinear Schwarzschild black holes. It is seen that the black holes' horizons are deformed by their mutual gravitational attraction. The geometry has a string like conical singularity that connects the holes but has nevertheless a well defined action. Using standard gravitational thermodynamics techniques we determine the Free energy for two black holes at fixed temperature and distance, their entropy and mutual force. When the black holes are far apart the results agree with Newtonian gravity expectations. This analyses is generalized to the case of charged black holes. Then we consider black holes embedded in String/Mtheory as bound states of branes. Using the effective string description of these bound states and for large separation we reproduce exactly the semi-classical result for the entropy, including the correction associated with the interaction between the holes.
\end{abstract}

\footnotetext{
${ }^{1}$ miguel@feynman.princeton.edu

${ }^{2}$ malcolm@damtp.cam.ac.uk
} 


\section{Introduction}

A longstanding and poorly explored problem in General Relativity is the interaction between Schwarzschild black holes, or more generally the interaction between ReissnerNordstrom black holes. While extremal Reissner-Nordstrom black holes are in equilibrium, corresponding in the modern language to BPS states of an yet unknown supersymmetric theory, in the non-extremal case the holes are expected to attract each other. It may then come as a surprise that a static solution representing $l$ Schwarzschild black holes placed along a common axis is indeed known in the literature [1]. The aim of this paper is to study such holes by using standard gravity techniques as well as the modern String Theory approach to black hole physics.

The geometry representing $l$ collinear Schwarzschild black holes has been known for a while [1]. It belongs to the general class of static axisymmetric Einstein vacua studied by Weyl [2], who showed that the problem of finding such solutions can be reduced to an associated problem in Newtonian gravity. In the case of $l$ collinear Schwarzschild black holes of mass $M_{n}$ each, one needs to find the classical potential for a system of $l$ rods each with mass $M_{n}$ and length $2 M_{n} G$. It may appear that for more than one hole this solution represents $l$ Schwarzschild black holes in equilibrium. However, such a solution fails the elementary flatness requirement [3]. In other words, the geometry contains conical singularities (similar to those found for cosmic strings) on the sections between the holes. The failure to find a spacetime free of singularities is interpreted as an instability, i.e. two of such black holes cannot be in static equilibrium [4, 5]. This fact may seem discouraging, however the above solution, and in particular the contribution from the conical singularities, still has a well defined gravitational action [6]. This means that we can use standard Euclidean Gravity thermodynamical arguments. The corresponding thermodynamical potential describes a system of black holes at fixed temperature and at fixed distance, i.e. we interpret the conical singularities as boundary conditions that keep the holes at fixed distance preventing the collapse of the system.

A similar analyses can be done for charged black holes. The motivation is that in some cases such holes have a natural embedding in String/M-theory as bound states of branes. Here we shall consider the triple intersection of M5-branes over a string [8, 9]. Remarkably, using the effective string description of these bound states and for large separation between the holes we were able to reproduce the entropy formula derived in the gravitational analysis. We find this fact reassuring, confirming that the old solution representing

collinear Schwarzschild black holes and the new charged ones are in fact sensible solutions, if interpreted appropriately. 
This paper starts in Section Two by reviewing the geometry representing $l$ collinear Schwarzschild black holes. Then we analyze the black holes' horizons, in particular how they are deformed by the mutual gravitational attraction between the holes. We shall see that the horizons area and surface gravity are changed by the presence of other holes. Then we discuss the conical singularity between the holes and its stringy description. The energy associated with such string is identified with the binding energy between the black holes and agrees with Newtonian gravity for large distances. In fact, we shall argue that for short distances, the gravitational approach breaks down because we are dealing with physics at the Planck scale. This happens when the holes are expected to merge. We proceed by determining the action for the Euclidean solution which is related to the Free energy for a system of black holes at fixed temperature and at fixed distance. We find that for large separation the entropy follows the area law and the generalized force between the holes is in agreement with Newtonian gravity.

Section Three is devoted to the study of charged black holes. First we embed the collinear Schwarzschild solution in five dimensions, apply a boost along the extra compact direction and reduce back to four dimensions. This procedure generates a solution representing $l$ collinear charged black hole in Einstein-Maxwell gravity with a scalar field. Then it is straightforward to find the solution with arbitrary $e^{a \phi} F^{2}$ coupling between the scalar field and the electromagnetic field. We analyze the properties of the charged black holes, with an emphasis on thermodynamics, generalizing the results mentioned above for uncharged black holes.

After finding the solution representing $l$ collinear non-extremal charged black holes we were lead to use String Theory to reproduce the results obtained in the semi-classical approach. This is the topic discussed in Section Four. We shall consider two black holes, each embedded in String/M-theory as a triple intersection of M5-branes over a string. Using the effective string description of such black holes we were able to reproduce exactly the corrections to the entropy due to the interaction between the holes. Furthermore, we resolve a puzzle that arose in the semi-classical calculation of the mean energy. Apparently the term associated with the interaction between the holes has the wrong sign to be associated with a Coulomb attractive potential. Careful considerations of the effective string model lead to the following solution of the problem: the left- and right-movers on the effective strings interact through an attractive Coulomb potential reducing their energy by $V_{\text {int }}$. As a consequence the left- and right-mover levels are shifted, leading to the correct entropy and energy formulae. Essentially the problem arises because Newtonian gravity ignores the black holes internal degrees of freedom, which are affected by this long range interaction.

We give our conclusions in Section Five. 


\section{Collinear Schwarzschild Black Holes}

Any static axisymmetric vacuum space-time, with two commuting killing vectors $\frac{\partial}{\partial t}$ and $\frac{\partial}{\partial \phi}$, possesses a metric that can be written in the form

$$
d s^{2}=-V d t^{2}+V^{-1} \gamma_{i j} d x^{i} d x^{j}
$$

with

$$
\gamma_{i j} d x^{i} d x^{j}=e^{2 K}\left(d \rho^{2}+d z^{2}\right)+\rho^{2} d \phi^{2} .
$$

The functions $V$ and $K$ depend on $\rho$ and $z$ only. With these assumptions the Einstein field equations read

$$
K_{\rho}=\frac{\rho}{4 V^{2}}\left(V_{\rho}^{2}-V_{z}^{2}\right), \quad K_{z}=\frac{\rho}{2 V^{2}} V_{\rho} V_{z},
$$

with the integrability condition $V \nabla^{2} V=\nabla V \cdot \nabla V$, where $\nabla$ is the taken with respect to flat Euclidean space in cylindrical coordinates. The subscripts in the functions $V$ and $K$ represent partial differentiation with respect to that variable. Defining a function $U$ by $V=e^{2 U}$ the equations of motion become

$$
K_{\rho}=\rho\left(U_{\rho}^{2}-U_{z}^{2}\right), \quad K_{z}=2 \rho U_{\rho} U_{z},
$$

with the integrability condition

$$
\nabla^{2} U=\left(\frac{\partial^{2}}{\partial \rho}+\frac{1}{\rho} \frac{\partial}{\partial \rho}+\frac{\partial^{2}}{\partial z^{2}}\right) U=0 .
$$

The function $U$ is formally the Newtonian potential outside some mass distribution. Thus the problem of finding the metric for a static axisymmetric space-time has been reduced to an associated problem in Newtonian gravity.

In the case of the Schwarzschild black hole $U$ is the potential for a rod of length $\mu=2 M G$ and mass $M$ (i.e. mass density $1 / 2 G$ ). The solution representing $l$ collinear Schwarzschild black holes is obtained by considering the potential for $l$ rods of length $\mu_{n}=2 M_{n} G$ and mass $M_{n}$ [1]:

$$
\begin{gathered}
U=\frac{1}{2} \sum_{n=1}^{l} \ln \frac{r_{n}^{+}+r_{n}^{-}-\mu_{n}}{r_{n}^{+}+r_{n}^{-}+\mu_{n}}, \\
K=\frac{1}{4} \sum_{n, m=1}^{l} \ln \left(\frac{r_{n}^{+} r_{m}^{-}+\left[z-\left(z_{n}+\mu_{n} / 2\right)\right]\left[z-\left(z_{m}-\mu_{m} / 2\right)\right]+\rho^{2}}{r_{n}^{+} r_{m}^{+}+\left[z-\left(z_{n}+\mu_{n} / 2\right)\right]\left[z-\left(z_{m}+\mu_{m} / 2\right)\right]+\rho^{2}}\right. \\
\left.\frac{r_{n}^{-} r_{m}^{+}+\left[z-\left(z_{n}-\mu_{n} / 2\right)\right]\left[z-\left(z_{m}+\mu_{m} / 2\right)\right]+\rho^{2}}{r_{n}^{-} r_{m}^{-}+\left[z-\left(z_{n}-\mu_{n} / 2\right)\right]\left[z-\left(z_{m}-\mu_{m} / 2\right)\right]+\rho^{2}}\right),
\end{gathered}
$$




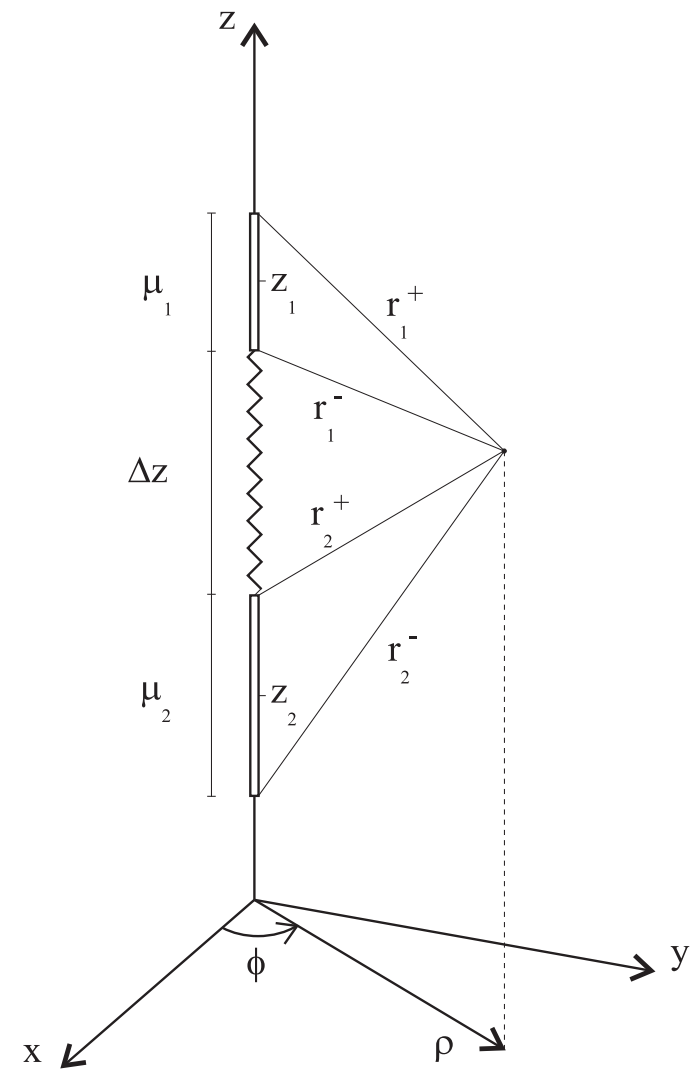

Figure 1: The case with two Schwarzschild black holes. Each rod is the locus for the holes' horizon and the section between the rods has a conical singularity. The Newtonian distance between the rods is $\Delta z=z_{1}-z_{2}-\left(\mu_{1}+\mu_{2}\right) / 2$.

where $z_{n}$ is the $n$-th rod mid point and $r_{n}^{+}$and $r_{n}^{-}$are given by

$$
r_{n}^{ \pm}=\sqrt{\rho^{2}+\left[z-\left(z_{n} \pm \mu_{n} / 2\right)\right]} .
$$

For simplicity we shall consider the case with two black holes but the results here presented are easily generalized to an arbitrary number of holes. In Figure 1 we represent the rods in the coordinate system used so far. Notice that in the single rod case the change of coordinates

$$
\rho=r \sqrt{1-\frac{\mu_{1}}{r}} \sin \theta, \quad z-z_{1}=\left(r-\mu_{1} / 2\right) \cos \theta .
$$

brings the metric to the usual form known for the Schwarzschild black hole.

Let us start by determining the ADM mass associated with this background. From the asymptotic behavior

$$
V \sim 1-\frac{\mu_{1}+\mu_{2}}{r}+\mathcal{O}\left(\frac{1}{r^{2}}\right), \quad K \sim \frac{1}{4} \ln \left(1+\mathcal{O}\left(\frac{1}{r^{2}}\right)\right) \sim \mathcal{O}\left(\frac{1}{r^{2}}\right),
$$


we conclude that the mass is $M_{1}+M_{2}$ as expected. Asymptotically space-time is flat and, provided the polar angle $\phi$ is defined modulo $2 \pi$, free of any singularity.

Next consider the metric near the $z$ axis. Along the sections that extend from the rods to infinity we have $K=0$ and $V$ has a well behaved limit. Hence the metric is well behaved along these sections of the $z$ axis. The holes' horizons correspond to the sections with rods. We postpone a detail discussion of the horizons to the next subsection. Along the section between both rods the function $K$ is

$$
K=\ln \left(\frac{\Delta z+\mu_{1}+\mu_{2}}{\Delta z+\mu_{1}} \frac{\Delta z}{\Delta z+\mu_{2}}\right),
$$

and $V$ has a well behaved limit as well. Inspecting the spatial part of the metric given by equation (2.2) we conclude that there is a conical singularity connecting both rods. The deficit angle $\delta$ satisfies

$$
\frac{\delta}{2 \pi}=\left(1-e^{-K}\right)=-\frac{\mu_{1} \mu_{2}}{\left(\Delta z+\mu_{1}+\mu_{2}\right) \Delta z} .
$$

In fact $\delta$ is negative so we have an excess angle. We conclude that the solution representing two Schwarzschild black holes is not free of singularities. This conical singularity signals an instability: two Schwarzschild black holes cannot be in equilibrium [四, 可. This fact has discouraged further work on the subject over the years. However, it was pointed out in [6] that this solution has a well defined gravitational action. Essentially this means that we can do gravitational thermodynamics and, if correctly interpreting the conical singularity, extract new results from an apparently ill defined geometry. We end here our review of collinear Schwarzschild black holes. Further details can be found in references [1], 因, 5].

\subsection{Horizons}

To study the horizons we need to investigate the behavior of the metric near the rods. To be definite we shall consider the first rod corresponding to the black hole with mass $M_{1}$. Then performing the coordinate transformation (2.8) the metric near $r=\mu_{1}=2 M_{1} G$ takes the form

$$
\begin{gathered}
d s^{2} \sim f^{2}(\theta)\left[-\left(1-\frac{\mu_{1}}{r}\right) d t^{2}+\left(\frac{\Delta z+\mu_{1}+\mu_{2}}{\Delta z+\mu_{1}}\right)^{2}\left(1-\frac{\mu_{1}}{r}\right)^{-1} d r^{2}\right] \\
+\mu_{1}^{2}\left[\left(\frac{\Delta z+\mu_{1}+\mu_{2}}{\Delta z+\mu_{1}}\right)^{2} f^{2}(\theta) d \theta^{2}+f^{-2}(\theta) \sin ^{2} \theta d \phi^{2}\right]
\end{gathered}
$$


where

$$
f(\theta)=\sqrt{\frac{\Delta z+\mu_{1}(\cos \theta+1) / 2}{\Delta z+\mu_{1}(\cos \theta+1) / 2+\mu_{2}}} .
$$

Clearly the surface $r=\mu_{1}$ and $t=$ const. is a horizon with surface gravity and area given by

$$
\begin{gathered}
\kappa_{1}=\frac{1}{2 \mu_{1}} \frac{\Delta z+\mu_{1}}{\Delta z+\mu_{1}+\mu_{2}}, \\
A\left(H_{1}\right)=4 \pi \mu_{1}^{2} \frac{\Delta z+\mu_{1}+\mu_{2}}{\Delta z+\mu_{1}} .
\end{gathered}
$$

As usual the temperature of the black hole is related to the horizon surface gravity by $T_{1}=\kappa_{1} /(2 \pi)$. In the limit $\Delta z=0$, we have $\kappa^{-1}=2\left(\mu_{1}+\mu_{2}\right)$ and $A\left(H_{1}+H_{2}\right)=4 \pi\left(\mu_{1}+\mu_{2}\right)^{2}$. This limit corresponds to the case when both rods come together forming a single rod with mass $M_{1}+M_{2}$ and therefore a single black hole. On the other hand in the limit $\Delta z \rightarrow \infty$, we have $\kappa_{n}{ }^{-1}=2 \mu_{n}$ and $A\left(H_{n}\right)=4 \pi \mu_{n}^{2}$. This is expected because when both holes are far apart each one should behave as in the single black hole case.

We shall be interested in the study of interacting black holes in thermal equilibrium. Equating the holes' temperature and discarding the trivial $\Delta z=0$ case we must have $M_{1}=M_{2} \equiv M$ ( or $\mu_{1}=\mu_{2} \equiv \mu$ ). We are led to the following picture. For large $\Delta z$ we have two holes with mass $M$ and temperature $T=(8 \pi M G)^{-1}$. The horizons are almost spherical and the holes are connected by a conical singularity. As $\Delta z$ decreases we expect the horizons to be deformed to a egg like shape, with the conical singularity that connects both holes emanating from the flat sides of the horizons. The temperature decreases. When $\Delta z=0$ the horizons become the North and South hemispheres of a single black hole horizon with a mass equal to $2 M$.

To make the above claim precise consider the angular part of the metric near the first rod

$$
d s_{\text {ang }}^{2} \sim \mu^{2}\left[A^{2} f^{2}(\theta) d \theta^{2}+f^{-2}(\theta) \sin ^{2} \theta d \phi^{2}\right]
$$

with

$$
A=\frac{\Delta z+2 \mu}{\Delta z+\mu}, \quad f(\theta)=\sqrt{\frac{\Delta z+\mu(\cos \theta+1) / 2}{\Delta z+\mu(\cos \theta+3) / 2}} .
$$

Next change to the angular variable $\tilde{\theta}=\tilde{\theta}(\theta)$ by requiring

$$
d s_{\text {ang }}^{2} \sim \mu^{2} g^{2}(\tilde{\theta})\left[d \tilde{\theta}^{2}+\sin ^{2} \tilde{\theta} d \phi^{2}\right] .
$$

Then $\tilde{\theta}=\tilde{\theta}(\theta)$ and $g(\tilde{\theta})$ are uniquely determined by

$$
\int \frac{d \tilde{\theta}}{\sin \tilde{\theta}}=A \int f^{2}(\theta) \frac{d \theta}{\sin \theta}, \quad g(\tilde{\theta}) \sin \tilde{\theta}=\frac{\sin \theta}{f(\theta)} .
$$




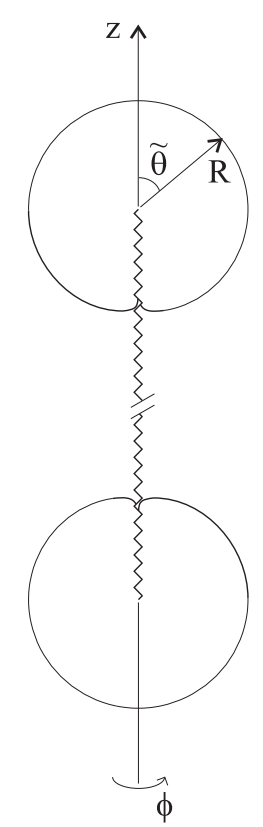

(a)

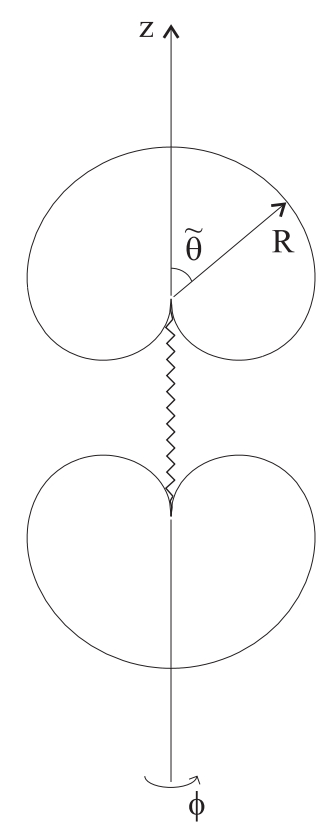

(b)

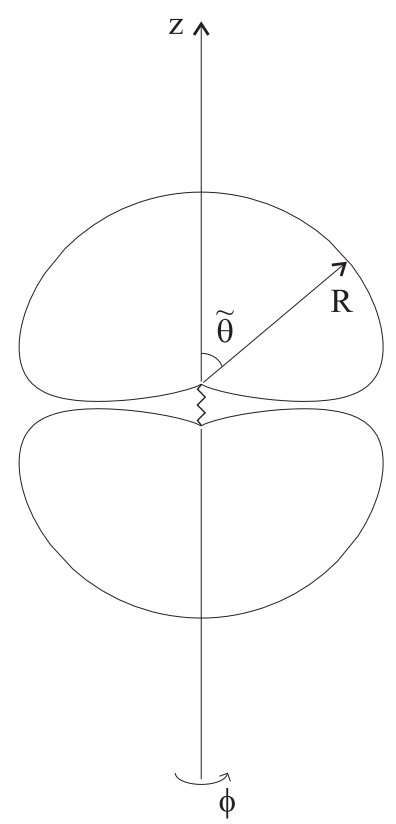

(c)

Figure 2: The horizons of the black holes $R=R(\tilde{\theta})$ for different values of the distance parameter $\alpha=2 \Delta z / \mu$. For (a) large separation $\alpha=10$ the horizons are almost spherical with radius $R \sim 2 M G$. The proper distance between the holes is $L \sim(1.83) \Delta z$. For (b) $\alpha=1$ the horizons are quite deformed and $L \sim(4.32) \Delta z$. For (c) small separation $\alpha=0.1$ each horizon becomes a hemisphere with radius $R \sim 4 M G$ and $L \sim(5.99) \Delta z$.

The integral can be done explicitly with the result

$$
\tan (\tilde{\theta} / 2)=C \tan (\theta / 2)(\cos (\theta / 2))^{\frac{4}{(\alpha+2)^{2}}}(3+\cos \theta+\alpha)^{-\frac{2}{(\alpha+2)^{2}}},
$$

where $C$ an integration constant and $\alpha=2 \Delta z / \mu$. The metric (2.17) defines an axisymmetric two-surface with radius $R(\tilde{\theta})=\mu g(\tilde{\theta})$. The constant $C$ can be fixed by requiring $g^{\prime}(0)=0$, i.e. the surface is regular at the pole opposite to the other hole. The result is

$$
C^{2}=\frac{\alpha^{2}+6 \alpha+4}{\alpha+2}(\alpha+4)^{\frac{4}{(\alpha+2)^{2}}-1} .
$$

As a check notice that for $\Delta z=0, C=\sqrt{2}$ and $\tan (\tilde{\theta} / 2)=\sqrt{2} \sin (\theta / 2) / \sqrt{3+\cos \theta}$. In this case $\tilde{\theta} \in[0, \pi / 2]$ and the two-surface is a North hemisphere with radius $2 \mu$ as pointed out before. When $\Delta z \rightarrow \infty$ we have $C=1, \tilde{\theta}=\theta$ and the two-surface becomes a twosphere with radius $\mu$. In Figure 2 the horizons are represented for the cases $\alpha=10,1,0.1$.

Finally we investigate the relation between the proper length $L$ of the section with a conical singularity, i.e. the distance between the singular points in the holes' horizons, and 
the distance $\Delta z$ in the associated Newtonian problem. A short calculation shows that

$$
L=e^{K} \int_{z_{2}+\mu / 2}^{z_{1}-\mu / 2} \frac{d z}{\sqrt{V}}=\Delta z\left(\frac{\alpha+4}{\alpha+2}\right)^{2} E(m), \quad m=\left(\frac{\alpha}{\alpha+4}\right)^{2},
$$

where $E(m)$ is the Complete Elliptic Integral of the Second Kind. Indeed only for large distances between the holes we have $L \sim \Delta z$ (recall that $E(1)=1$ ). For small distances we have $L \sim 2 \pi \Delta z$ (recall that $E(0)=\pi / 2$ ).

\subsection{Conical Singularity}

Let us start by writing the spacetime metric along the section between both rods

$$
d s^{2} \sim\left(-V(z) d t^{2}+V^{-1}(z) e^{2 K} d z^{2}\right)+V^{-1}(z)\left(e^{2 K} d \rho^{2}+\rho^{2} d \phi^{2}\right)
$$

where

$$
V(z)=\frac{z-\left(z_{1}-\mu / 2\right)}{z-\left(z_{1}+\mu / 2\right)} \frac{z-\left(z_{2}+\mu / 2\right)}{z-\left(z_{2}-\mu / 2\right)}, \quad e^{K}=\frac{(\Delta z+2 \mu) \Delta z}{(\Delta z+\mu)^{2}} .
$$

The deficit angle along the axis is

$$
\frac{\delta}{2 \pi}=-\frac{\mu^{2}}{(\Delta z+2 \mu) \Delta z} .
$$

This geometry is Ricci flat, however the presence of a conical singularity on the axis means that [0]

$$
\frac{1}{2} \int d^{4} x \sqrt{-g} R=\text { Area } \cdot \delta
$$

where Area is the space-time area of the surface spanned by the conical singularity. It follows that the Ricci scalar can be defined distributionally according to

$$
R=-V(z) \frac{4 \pi \mu^{2}}{(\Delta z+2 \mu) \Delta z} \delta(x, y)
$$

where $(x, y)$ are the cartesian coordinates in the plane orthogonal to the $z$ axis. Furthermore, since the four-dimensional metric factorizes and this plane is a two-dimensional manifold we can use the result $R_{a b}=(R / 2) g_{a b}$ to determine the non-zero components of the Ricci tensor: $R_{x x}=R_{y y}=R / 2$. This defines, by means of the Einstein field equations $G_{\mu \nu}=8 \pi G T_{\mu \nu}$, an effective energy-momentum tensor associated with the conical singularity:

$$
T^{\mu \nu}=-\frac{\mu^{2}}{4 G(\Delta z+2 \mu) \Delta z} \delta(x, y) \operatorname{diag}\left(1,0,0,-V^{2} e^{-2 K}\right)
$$


The situation is similar to the case of cosmic strings, but with the important difference that the deficit angle and therefore the tension $T=-\mu^{2} /(4 G(\Delta z+2 \mu) \Delta z)$ are negative. The singularity is therefore usually referred to as a strut rather than a string.

To determine the energy associated with this strut, let $v=\frac{\partial}{\partial t}$ and define the unit vector $\zeta$ by $\zeta=v /(-v \cdot v)^{1 / 2}$. Then the energy density as seen by a static observer placed at infinity is

$$
T^{\mu \nu} v_{\mu} \zeta_{\nu}=-\frac{\mu^{2} V^{3 / 2}(z)}{4 G(\Delta z+2 \mu) \Delta z} \delta(x, y)
$$

The total energy associated with the strut as seen by this observer is obtained by integrating over the spatial directions

$$
E=-\frac{\mu^{2} \Delta z}{4 G(\Delta z+\mu)^{2}} .
$$

This is interpreted as the gravitational binding energy between both black holes. In fact, for $\mu / \Delta z \ll 1$ we have $E \sim-M^{2} G / \Delta z$, the Newtonian potential between two particles of $\operatorname{mass} M$.

Next let $w=\frac{\partial}{\partial z}$ and define the unit vector $\lambda$ by $\lambda=w /(w \cdot w)^{1 / 2}$. The pressure along the $z$ axis is

$$
T^{\mu \nu} \lambda_{\mu} \lambda_{\nu}=\frac{\mu^{2} V(z)}{4 G(\Delta z+2 \mu) \Delta z} \delta(x, y),
$$

which equals minus the local energy density. The pressure on the strut is obtained by integrating over the $x-y$ plane:

$$
P=\frac{\mu^{2}}{4 G(\Delta z+2 \mu) \Delta z},
$$

which for $\mu / \Delta z \ll 1$ gives $P \sim M^{2} G / \Delta z^{2}$. This explains why the black holes are kept at a fixed distance since the pressure exerted on the holes will cancel the gravitational attraction.

To understand the physics underlying this problem, notice that this geometry was found by requiring the coordinate distance $\Delta z$ to be fixed. In fact, in the associated Newtonian problem we considered the potential for two rods separated by a distance $\Delta z$ but the gravitational attraction between the rods was ignored. Certainly the black holes will attract each other and are not in equilibrium. This is the reason why requiring $\Delta z$ fixed leads to a conical singularity connecting both holes. Effectively this conical singularity can be seen as a strut connecting both holes. The strut energy is interpreted as the interaction energy between the black holes while its pressure prevents the gravitational collapse of the system. For large distances between the holes this interpretation is consistent with Newtonian gravity. Hence the presence of the conical singularity is in agreement with the energetics of the problem and with keeping $\Delta z$ fixed. 


\subsection{Gravitational Thermodynamics}

The novelty of this solution is that its action is well defined. Therefore by the usual semiclassical gravitational thermodynamics arguments we can calculate the Free energy for two Schwarzschild black holes at fixed temperature and distance. This defines the entropy of the system and the generalized force conjugate to $\Delta z$, which for large distances should agree with the Newton force between two particles of mass $M$. Furthermore, the system will evolve such that the minimum of the thermodynamical potential is attained.

Before we start this calculation it is important to analyze whether the semi-classical evaluation of the partition function is reliable. We expect the General Relativity description to be valid for small deficit angle $|\delta|$. Effectively this corresponds to have a strut tension well below the Planck scale:

$$
\frac{\mu^{2}}{4 G(\Delta z+2 \mu) \Delta z} \ll \frac{1}{G} \Rightarrow \frac{\mu}{\Delta z} \ll 1,
$$

which happens when the black holes are at a large proper distance from each other. Thus this work will focus on well separated black holes interacting through a Coulomb potential. This corresponds to the limit where both holes' horizons are nearly spherical as represented in Figure 2(a).

The Euclidean action for the solution is

$$
I=\beta\left(2 \frac{M}{2}+\frac{M^{2} G \Delta z}{(\Delta z+2 M G)^{2}}\right)=\beta\left(\frac{\mu}{2 G}+\frac{\mu^{2} \Delta z}{4 G(\Delta z+\mu)^{2}}\right),
$$

where

$$
\frac{1}{T}=\beta=4 \pi \mu \frac{\Delta z+2 \mu}{\Delta z+\mu},
$$

is the Euclidean time periodicity. The metric is regular at the fixed point sets of the Euclidean time translation Killing vector $\frac{\partial}{\partial \tau}$, that correspond to the two horizons in the Lorentzian version. These bolts are connected by the conical singularity along which $\frac{\partial}{\partial \tau}$ has no fixed points.

From (2.33) we can read the thermodynamical potential for two Schwarzschild black holes at fixed temperature and distance evaluated in the semi-classical limit:

$$
\mathcal{F}(T, \Delta z)=\frac{\mu}{2 G}+\frac{\mu^{2} \Delta z}{4 G(\Delta z+\mu)^{2}},
$$

where $\mu=\mu(T, \Delta z)$ through the relation (2.34). Then the entropy and generalized force conjugate to $\Delta z$ are

$$
S=-\left.\frac{\partial \mathcal{F}}{\partial T}\right|_{\Delta z}, \quad F_{\Delta z}=-\left.\frac{\partial \mathcal{F}}{\partial \Delta z}\right|_{T}
$$


It is useful to determine first $\left.\frac{\partial \mu}{\partial T}\right|_{\Delta z}$ and $\left.\frac{\partial \mu}{\partial \Delta z}\right|_{T}$. The result is

$$
\begin{gathered}
\left.\frac{\partial \mu}{\partial T}\right|_{\Delta z}=-4 \pi \mu^{2} \frac{(\Delta z+2 \mu)^{2}}{\Delta z^{2}+4 \mu \Delta z+2 \mu^{2}}=-4 \pi \mu^{2}\left[1+\mathcal{O}\left(\frac{\mu}{\Delta z}\right)^{2}\right], \\
\left.\frac{\partial \mu}{\partial \Delta z}\right|_{T}=\frac{\mu^{2}}{\Delta z^{2}+4 \mu \Delta z+2 \mu^{2}}=\frac{\mu^{2}}{\Delta z^{2}}\left[1+\mathcal{O}\left(\frac{\mu}{\Delta z}\right)\right] .
\end{gathered}
$$

Then the entropy and generalized force are

$$
\begin{gathered}
S=8 \pi G M^{2}\left[1+\frac{\mu}{\Delta z}+\mathcal{O}\left(\frac{\mu}{\Delta z}\right)^{2}\right]=\frac{1}{4 G}\left(A\left(H_{1}\right)+A\left(H_{2}\right)\right) \\
F_{\Delta z}=-\frac{M^{2} G}{\Delta z^{2}}\left[1+\mathcal{O}\left(\frac{\mu}{\Delta z}\right)\right] .
\end{gathered}
$$

To order $\mu / \Delta z$ the entropy satisfies the area law. The correction arises from the conical singularity contribution to the Free energy and it is associated to the deformation of the horizon due to the gravitational attraction between the holes. The generalized force $F_{\Delta z}$ matches exactly the Newtonian force between two particles of mass $M$, as expected for largely separated black holes.

In spite of the fact that the above results seem consistent with Newtonian gravity it may look surprising that for large distances the second term in the Free energy (2.35) has the wrong sign to be interpreted as the Free energy for two gravitating particles of mass $M$. The same comment applies to the mean value of the energy

$$
E=\mathcal{F}+T S=2 M+\frac{M^{2} G}{\Delta z}\left[1+\mathcal{O}\left(\frac{\mu}{\Delta z}\right)\right] .
$$

Firstly, notice that the Free energy decreases as $\Delta z$ decreases because $M$ is not fixed. Figure 3 shows a plot of the Free energy for fixed temperature as $\Delta z$ varies. The minimum of the thermodynamical potential corresponds to the single black hole case while for large distances the holes will tend to attract each other. Secondly, the apparently wrong signs in (2.35) and (2.39) may be telling us that there are other internal degrees of freedom in the black holes that are being excited due to the gravitational interaction, with this net result. With the help of String Theory we shall see in Section Four that this is indeed the case. In fact, only by fixing the internal degrees of freedom of the black holes we should expect the variation of the energy to follow the Newtonian gravity prediction. To see this consider the energy variation under an adiabatic transformation

$$
d E=T d S-F_{\Delta z} d(\Delta z)
$$




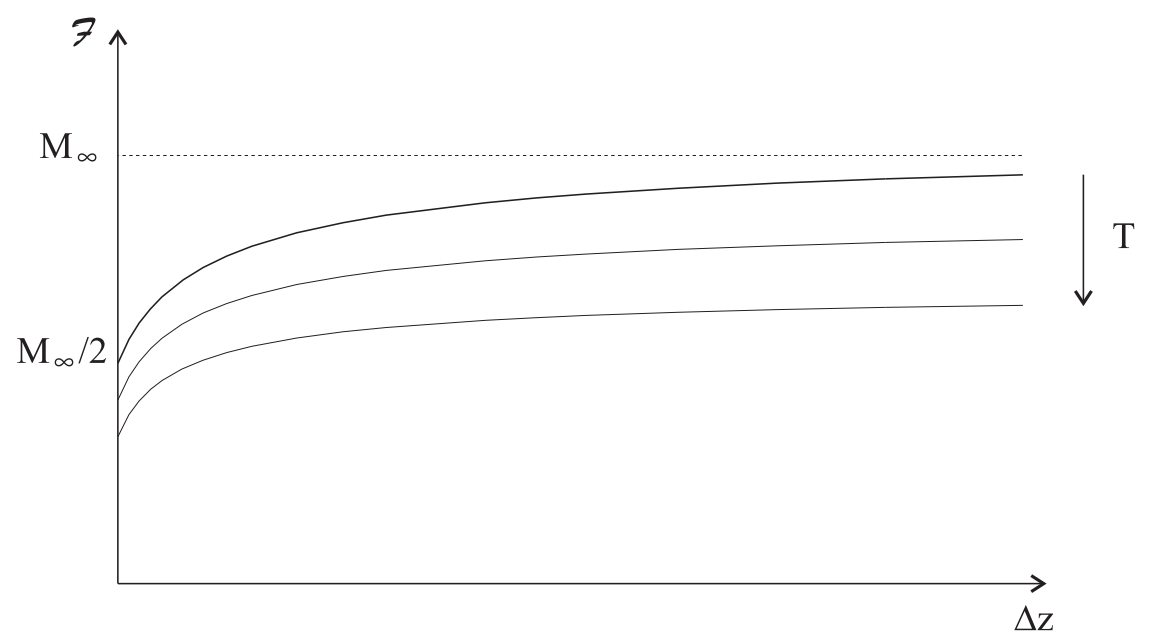

Figure 3: The Free Energy as a function of the distance parameter $\Delta z$ for fixed temperature. The mass of the holes when they are far apart is related to the temperature by $M_{\infty}=(8 \pi G T)^{-1}$. For $\Delta z=\infty$ we have two holes of mass $M_{\infty}$ and a Free Energy $\mathcal{F}=2\left(M_{\infty} / 2\right)$, while for $\Delta z=0$ we have one hole with the same mass and a Free Energy $\mathcal{F}=M_{\infty} / 2$. To minimize the thermodynamical potential the holes tend to decrease their distance (and to increase the temperature as usual for Schwarzschild black holes).

For a reversible process, i.e. at fixed entropy keeping the number of internal degrees of freedom fixed, we have

$$
d E=\frac{M^{2} G}{\Delta z^{2}} d(\Delta z) \Rightarrow E_{f}-E_{i}=-\left.\frac{M^{2} G}{\Delta z}\right|_{\Delta z_{i}} ^{\Delta z_{f}}
$$

in agreement with Newtonian gravity.

\section{Charged Black Holes}

The goal of this section is to generalize the previous results to charged black holes. First consider the five-dimensional Einstein theory and compactify the fifth direction on a circle according to

$$
d s_{5}^{2}=e^{-\frac{2}{\sqrt{3}} \phi}\left(d y-A_{\mu} d x^{\mu}\right)^{2}+e^{\frac{1}{\sqrt{3}} \phi} g_{\mu \nu} d x^{\mu} d x^{\nu} .
$$

Then the action for the four-dimensional theory reads

$$
I=\frac{1}{16 \pi G} \int d^{4} x \sqrt{-g}\left[R-\frac{1}{2}(\partial \phi)^{2}-\frac{1}{4} e^{-\sqrt{3} \phi} F^{2}\right],
$$


where $F=d A$. Next embed the solution representing $l$ collinear Schwarzschild black holes (2.1) in five dimensions by adding an extra flat direction

$$
d s_{5}^{2}=-V d t^{2}+V^{-1} \gamma_{i j} d x^{i} d x^{j}+d y^{2}
$$

where $\gamma_{i j}$ is given by (2.2) and the functions $V=e^{2 U}$ and $K$ are as in (2.6). Applying a boost along the $y$ direction the metric becomes

$$
\begin{aligned}
d s_{5}{ }^{2}= & -\frac{V}{\cosh ^{2} \sigma-V \sinh ^{2} \sigma} d t^{2}+V^{-1} \gamma_{i j} d x^{i} d x^{j} \\
& +\left(\cosh ^{2} \sigma-V \sinh ^{2} \sigma\right)\left(d y-\frac{(1-V) \cosh \sigma \sinh \sigma}{\cosh ^{2} \sigma-V \sinh ^{2} \sigma} d t\right)^{2} .
\end{aligned}
$$

According to the ansatz (3.1) this corresponds to the following solution of the fourdimensional theory

$$
\begin{gathered}
d s^{2}=-H^{-\frac{1}{2}} V d t^{2}+H^{\frac{1}{2}} V^{-1} \gamma_{i j} d x^{i} d x^{j} \\
F=-\operatorname{coth} \sigma d\left(H^{-1}\right) \wedge d t, \quad e^{-\frac{2}{\sqrt{3}} \phi}=H
\end{gathered}
$$

where $H=1+\sinh ^{2} \sigma(1-V)$.

The above Kałuża-Klein solution can be generalized to an arbitrary $e^{a \phi} F^{2}$ coupling of the scalar field to the gauge field. The action

$$
I=\frac{1}{16 \pi G} \int d^{4} x \sqrt{-g}\left[R-\frac{1}{2}(\partial \phi)^{2}-\frac{1}{4} e^{a \phi} F^{2}\right],
$$

has the following solution representing $l$ collinear electrically charged black hole

$$
\begin{gathered}
d s^{2}=H^{\frac{2}{1+a^{2}}}\left[-\frac{V}{H^{\frac{4}{1+a^{2}}}} d t^{2}+V^{-1} \gamma_{i j} d x^{i} d x^{j}\right], \\
F=-\frac{2}{\sqrt{1+a^{2}}} \operatorname{coth} \sigma d\left(H^{-1}\right) \wedge d t, \quad e^{2 \phi}=H^{\frac{4 a}{1+a^{2}}} .
\end{gathered}
$$

The dual magnetic solution can be found by transforming $\phi \rightarrow-\phi$ and $F \rightarrow \star F$. For the sake of clarity we shall consider only electrically charged black holes.

From the asymptotics of this solution we can determine the ADM mass, the electric charge and the charge associated with the scalar field:

$$
\begin{gathered}
M_{\text {total }}=\sum_{n=1}^{l} M_{n}=\frac{1}{2 G}\left(1+\frac{2}{1+a^{2}} \sinh ^{2} \sigma\right) \sum_{n=1}^{l} \mu_{n} \\
Q_{\text {total }}=\sum_{n=1}^{l} Q_{n}=\frac{1}{\sqrt{1+a^{2}}} \sinh (2 \sigma) \sum_{n=1}^{l} \mu_{n} \\
\Sigma_{\text {total }}=\sum_{n=1}^{l} \Sigma_{n}=\frac{2 a}{1+a^{2}} \sinh ^{2} \sigma \sum_{n=1}^{l} \mu_{n} .
\end{gathered}
$$


The mass and charges of the $n$-th black hole together with the non-extremality parameter $\mu_{n}$ satisfy the important relation

$$
\mu_{n}^{2}=\left(2 M_{n} G+a \Sigma_{n}\right)^{2}-\left(1+a^{2}\right) Q_{n}^{2} .
$$

As usual the extremal limit corresponds to sending $\mu_{n} \rightarrow 0, \sigma \rightarrow \infty$ such that the charge $Q_{n}$ is kept fixed. Then $V=1, K=0$ and $H$ is a harmonic function.

For simplicity consider the solution representing two black holes. As before the rods correspond to the holes' horizons. Along the first rod the metric has the following limiting behavior:

$$
\begin{gathered}
d s^{2} \sim \cosh ^{\frac{4}{1+a^{2}}} \sigma\left\{f^{2}(\theta)\left[-\left(1-\frac{\mu_{1}}{r}\right) \frac{d t^{2}}{\cosh ^{\frac{8}{1+a^{2}}} \sigma}+\left(\frac{\Delta z+\mu_{1}+\mu_{2}}{\Delta z+\mu_{1}}\right)^{2}\left(1-\frac{\mu_{1}}{r}\right)^{-1} d r^{2}\right]\right. \\
\left.+\mu_{1}^{2}\left[\left(\frac{\Delta z+\mu_{1}+\mu_{2}}{\Delta z+\mu_{1}}\right)^{2} f^{2}(\theta) d \theta^{2}+f^{-2}(\theta) \sin ^{2} \theta d \phi^{2}\right]\right\},
\end{gathered}
$$

where

$$
f(\theta)=\sqrt{\frac{\Delta z+\mu_{1}(\cos \theta+1) / 2}{\Delta z+\mu_{1}(\cos \theta+1) / 2+\mu_{2}}} .
$$

The horizon surface gravity and area read

$$
\begin{gathered}
\kappa_{1}=\frac{1}{2 \mu_{1} \cosh ^{\frac{4}{1+a^{2}}} \sigma} \frac{\Delta z+\mu_{1}}{\Delta z+\mu_{1}+\mu_{2}}, \\
A\left(H_{1}\right)=4 \pi \mu_{1}^{2} \cosh ^{\frac{4}{1+a^{2}}} \sigma \frac{\Delta z+\mu_{1}+\mu_{2}}{\Delta z+\mu_{1}} .
\end{gathered}
$$

Again we shall consider black holes with the same temperature $T=\kappa /(2 \pi)$. Hence we require $\mu \equiv \mu_{1}=\mu_{2}$ and each hole has mass $M$ and electric charge $Q$. The analyses of the holes' horizons is entirely similar to the Schwarzschild case. The only difference is the conformal factor in the near-horizon metric (3.10).

Between the rods we have a conical singularity with deficit angle

$$
\frac{\delta}{2 \pi}=-\frac{\mu^{2}}{(\Delta z+2 \mu) \Delta z} .
$$

As before this conical singularity can be effectively described as a strut that connects both black holes. The corresponding energy as seen by a static observer at infinity and the pressure on the strut along the $z$ axis are

$$
E=-\frac{\mu^{2} \Delta z}{4 G(\Delta z+\mu)^{2}} \sim-\frac{\mu^{2}}{4 G \Delta z}, \quad P=\frac{\mu^{2}}{4 G(\Delta z+2 \mu) \Delta z} \sim \frac{\mu^{2}}{4 G \Delta z^{2}},
$$


respectively. The interpretation is similar to the Schwarzschild black holes case but now the holes interact through the gravitational, scalar and gauge fields exchange. This fact is revealed by formula (3.9) for the non-extremality parameter $\mu$.

\subsection{Gravitational Thermodynamics}

Our starting point is the Euclidean action for the solution representing two non-extremal charged black holes

$$
I=\beta\left(2 \frac{M}{2}-2 \frac{Q}{8 G} \Phi+\frac{\mu^{2} \Delta z}{4 G(\Delta z+\mu)^{2}}\right)=\beta\left(\frac{\mu}{2 G}+\frac{\mu^{2} \Delta z}{4 G(\Delta z+\mu)^{2}}\right),
$$

where

$$
\frac{1}{T}=\beta=4 \pi \mu \cosh ^{\frac{4}{1+a^{2}}} \sigma \frac{\Delta z+2 \mu}{\Delta z+\mu}, \quad \Phi=\frac{2}{\sqrt{1+a^{2}}} \tanh \sigma,
$$

are the Euclidean time periodicity and the horizons electric potential, respectively. Then the thermodynamical potential $W=E-T S-\frac{Q_{t o t a l}}{4 G} \Phi$ reads

$$
W(T, \Phi, \Delta z)=\frac{\mu}{2 G}+\frac{\mu^{2} \Delta z}{4 G(\Delta z+\mu)^{2}},
$$

with the non-extremality parameter $\mu=\mu(T, \Phi, \Delta z)$ defined through the relations (3.16). The entropy $S$, generalized force $F_{\Delta z}$ and total charge $Q_{\text {total }}$ are determined by the usual thermodynamical relations

$$
S=-\left.\frac{\partial W}{\partial T}\right|_{\Phi, \Delta z}, \quad F_{\Delta z}=-\left.\frac{\partial W}{\partial \Delta z}\right|_{T, \Phi}, \quad \frac{Q_{\text {total }}}{4 G}=-\left.\frac{\partial W}{\partial \Phi}\right|_{T, \Delta z} .
$$

After some straightforward algebra we obtained the following results:

$$
\begin{gathered}
S=\frac{8 \pi \mu^{2}}{4 G} \cosh ^{\frac{4}{1+a^{2}}} \sigma\left[1+\frac{\mu}{\Delta z}+\mathcal{O}\left(\frac{\mu}{\Delta z}\right)^{2}\right]=\frac{1}{4 G}\left(A\left(H_{1}\right)+A\left(H_{2}\right)\right), \\
F_{\Delta z}=-\frac{1}{4 G}\left(\frac{\mu}{\Delta z}\right)^{2}\left[1+\mathcal{O}\left(\frac{\mu}{\Delta z}\right)\right] \\
Q_{\text {total }}=2 Q\left[1+\mathcal{O}\left(\frac{\mu}{\Delta z}\right)^{2}\right] .
\end{gathered}
$$

To order $\mu / \Delta z$ the entropy follows the area law, the force $F_{\Delta z}$ arises from the gravitational and scalar field attraction and electric repulsion between the holes and the charge $Q_{\text {total }}$ is the sum of the holes electric charge. Notice that we are using an ensemble with the 
temperature and electric potential held fixed. For example, this means that the total mass and charge are not fixed quantities. In fact for large separation we expect two black holes each with the same mass and charge as for the single black hole case $(\Delta z=0)$.

Finally, as for the Schwarzschild black holes, the thermodynamical potential $W$ and the mean energy $E$ have an apparently wrong sign in the interacting term. However, holding the entropy and charges fixed the variation of the energy does follow the expected Coulomb-like behavior. We shall come back to this point in the next section.

\section{Interacting Black Holes in String/M-theory}

To have a better understanding of the microscopic structure underlying the previous results we shall analyze the problem in String/M-theory. We consider four-dimensional black holes with four $U(1)$ charges. A convenient representation of such holes is the non-extremal Mtheory configuration $5 \perp 5 \perp 5$ of three M5-branes intersecting over a common string along which momentum flows [8, 9] (the M5-branes intersect pairwise over a 3-brane). There are three magnetic charges related to the number of M5-branes in three different hyperplanes and a electric charge which has a Kałuża-Klein origin. The four-dimensional Einstein metric for two of such black holes aligned along the $z$ axis is

$$
d s^{2}=-H^{-\frac{1}{2}} V d t^{2}+H^{\frac{1}{2}} V^{-1} \gamma_{i j} d x^{i} d x^{j}
$$

where

$$
H=\prod_{i=1}^{4}\left(1+\sinh ^{2} \sigma_{i}(1-V)\right)
$$

and the functions $V=e^{2 U}$ and $K$ are given by (2.6) with $l=2$. We shall set $\mu \equiv \mu_{1}=\mu_{2}$ such that the black holes have the same temperature. The M5-brane and Kałuża-Klein charges associated with each hole are

$$
Q_{i}=\frac{\mu}{2} \sinh \left(2 \sigma_{i}\right)
$$

Next consider the dilute gas regime [10] where the energy associated with the Kałuża-Klein excitations is much smaller than the M5-branes mass:

$$
\mu, \mu \sinh \sigma_{4} \ll \mu \sinh \sigma_{j}, \quad j=1,2,3 .
$$

In this limit anti-branes are suppressed and the number of M5-branes $N_{j}$ satisfies

$$
\frac{e^{2 \sigma_{1}}}{4} \mu=\frac{N_{1}}{L_{6} L_{7}}\left(\frac{\kappa_{11}}{4 \pi}\right)^{2 / 3}, \quad \frac{e^{2 \sigma_{2}}}{4} \mu=\frac{N_{2}}{L_{4} L_{5}}\left(\frac{\kappa_{11}}{4 \pi}\right)^{2 / 3}, \quad \frac{e^{2 \sigma_{3}}}{4} \mu=\frac{N_{3}}{L_{2} L_{3}}\left(\frac{\kappa_{11}}{4 \pi}\right)^{2 / 3},
$$


where the eleven-dimensional gravitational coupling satisfies $\kappa_{11}^{2}=V_{7} \kappa^{2}$, with $V_{7}$ the volume of the compact space. The first set of M5-branes has two compact orthogonal directions with length $L_{6}$ and $L_{7}$ and similarly for the other M5-branes. The mass of each black hole becomes

$$
M=\frac{1}{4 G}\left(Q_{1}+Q_{2}+Q_{3}\right)+\frac{\mu}{8 G} \cosh \left(2 \sigma_{4}\right) \equiv M_{B P S}+\delta M,
$$

where $M_{B P S}$ is the total M5-branes mass and $\delta M$ is the mass associated with the KałużaKlein excitations along the common direction with length $L_{1}$. We interpret $M_{B P S}$ as the ground state mass, while the Kałuża-Klein modes correspond to the excitations of the system. The total momentum carried along this compact direction is

$$
P=P_{L}-P_{R}=\frac{\mu}{8 G} \sinh \left(2 \sigma_{4}\right)
$$

In recent String Theory developments black holes are described by a dual conformal field theory [11, 12, 13. In some cases this is a $1+1$ conformal field theory, as in the original work [14], commonly referred as the effective string description of black holes [1519]. For the M-theory configuration described above the black hole is described by an effective string with length $L_{\text {eff }}=N_{1} N_{2} N_{3} L_{1}$ and central charge $c=6$ [8]. Then one matches the energy and the momentum carried by left- and right-movers on this string with $\delta M$ and $P$, respectively. As a result the entropy formula is recovered exactly. In the case at hand each black hole will still be described by an effective string. However, the interaction between both black holes means that the strings will interact with each other and that the level for left- and right-movers will change. To make these ideas precise we write the thermodynamical potential $W$ and the entropy of the system in the dilute gas regime and for large distance between the holes

$$
\begin{gathered}
W=\frac{\mu}{2 G}+\frac{\mu^{2}}{4 G \Delta z}, \\
S=\frac{8 \pi}{4 G} \sqrt{Q_{1} Q_{2} Q_{3}} \sqrt{\mu} \cosh \sigma_{4}\left(1+\frac{\mu}{\Delta z}\right) .
\end{gathered}
$$

Then the energy mean value $E=W+T S+\frac{Q_{t o t a l}}{4 G} \Phi$ reads

$$
E=2\left(M_{B P S}+\delta M+\frac{\mu^{2}}{4 G \Delta z}\right)-\frac{\mu^{2}}{4 G \Delta z} \equiv 2\left(M_{B P S}+\delta E\right)+V_{i n t},
$$

where $M_{B P S}$ and $\delta M$ are defined in (4.6). We see that in this case the increase in energy $\delta E$ does not coincide with the increase in the mass $\delta M$ of each hole. As we start filling 


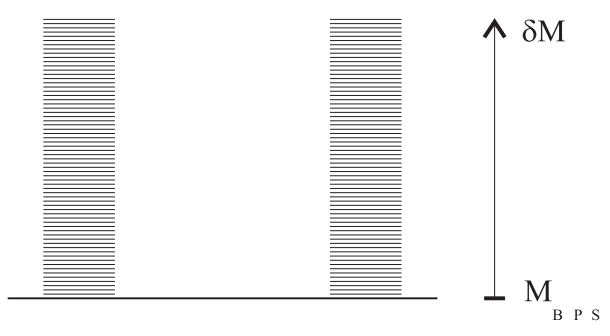

(a)

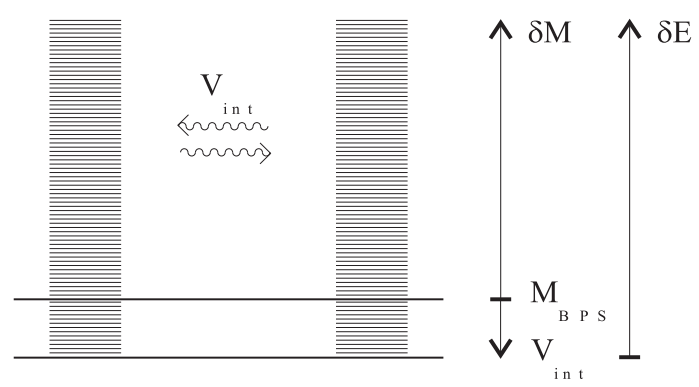

(b)

Figure 4: Representation of the tower of momentum states on the effective string model for both black holes when their mutual interaction is (a) switched off and (b) switched on. The effect of the Coulomb attraction on the effective string states is to shift their ground state energy by $V_{\text {int }}$.

the left- and right-moving states in each effective string, they will interact reducing their energy by $V_{\text {int }}$ according to a large distance attractive Coulomb potential. In fact, the dependence on the string coupling $V_{\text {int }} \sim-g^{2} N_{L} N_{R}$ suggests that in a similar D-brane setting (e.g. the $D 5-D 1$ system for five-dimensional black holes) the interactions arise from an annulus diagram with two insertions on a boundary. Thus, in order to reproduce a state corresponding to two black holes each of mass $M_{B P S}+\delta M$ we need an energy associated with the string excitations given by $\delta E=\delta M+\frac{\mu^{2}}{4 G \Delta z}$. This fact explains the apparent discrepancy in the sign of the interacting term in the thermodynamical potential $W$ and energy $E$. There are two different contributions: one from the Coulomb attraction and another from the black holes internal states. In Figure 4 we represent the effect of the interaction between the effective strings.

To determine the level of the left- and right-moving states on each effective string note that

$$
\begin{gathered}
\delta E=\frac{1}{4 G}\left[\frac{\mu \cosh 2 \sigma_{4}}{2}+\frac{\mu^{2}}{\Delta z}\right]=\frac{1}{4 G}\left[\left(\frac{e^{2 \sigma_{4}}}{4}+\frac{\mu}{2 \Delta z}\right) \mu+\left(\frac{e^{-2 \sigma_{4}}}{4}+\frac{\mu}{2 \Delta z}\right) \mu\right], \\
P=\frac{1}{4 G} \frac{\mu \sinh 2 \sigma_{4}}{2}=\frac{1}{4 G}\left[\left(\frac{e^{2 \sigma_{4}}}{4}+\frac{\mu}{2 \Delta z}\right) \mu-\left(\frac{e^{-2 \sigma_{4}}}{4}+\frac{\mu}{2 \Delta z}\right) \mu\right] .
\end{gathered}
$$

Hence there is a shift in the left- and right-mover levels. Now we have

$$
\kappa^{2} \frac{N_{L}}{L_{1}}=\left(\frac{e^{2 \sigma_{4}}}{4}+\frac{\mu}{2 \Delta z}\right) \mu, \quad \kappa^{2} \frac{N_{R}}{L_{1}}=\left(\frac{e^{-2 \sigma_{4}}}{4}+\frac{\mu}{2 \Delta z}\right) \mu .
$$

Then the entropy associated with both effective strings is

$$
S=2 \cdot 2 \pi \sqrt{N_{1} N_{2} N_{3}}\left(\sqrt{N_{L}}+\sqrt{N_{R}}\right)
$$


which matches exactly the result in (4.8), including the correction of order $\mu / \Delta z$. Thus we successfully reproduced the deviations in the entropy formula due to the long range interactions between these non-extremal charged black holes.

\section{Conclusion}

In this paper we analysed in detail the geometry representing $l$ collinear non-extremal black holes. In spite of the conical singularity connecting the black holes, this solution has a well defined action. This fact enabled us to study the interaction between largely separated non-extremal black holes by using standard gravitational thermodynamics techniques. Then we analysed this problem using the modern String Theory language and successfully reproduced the entropy formula for interacting black holes, including the correction due to the long range force between the holes. This interaction causes the left- and right-moving states that build up the internal degrees of freedom of the system to reduce their energy affecting the state counting.

It is interesting to note that the the binding energy due to the interaction between the holes is expected to lead to a energy shift in the observed Hawking radiation. In fact left- and right-movers with quantum numbers $n_{L, R}$ such that $\frac{n_{L, R}}{R_{1}}<-V_{\text {int }}$ are bounded and cannot leave the branes. Unfortunately, it may be very difficult to see this by solving Laplace equation (e.g. for a scalar field) in this background. For example, the same problem in the simpler double-centred extremal case turns out to be highly non-trivial 20, 21].

A point that is less clear to us is related to the quadratic and higher order corrections in $\mu / \Delta z$ to the thermodynamical quantities here calculated. In particular the entropy does not follow the area law to this order. We suspect that the conical singularity is a good approximation to the long range Coulomb interaction but breaks down otherwise. Indeed, we saw that for small distances between the black holes the physics is Planckian and our approximations are no longer valid.

A possible venue of research is the study of this geometry in the decoupling limit. This may lead to interesting results on the Coulomb branch of the dual field theory at finite temperature (which is not stable since the Coulomb branch is lifted). For example, a study of the $A d S / C F T$ duality for $\mathcal{N}=4$ Super-Yang-Mills at finite temperature using D3-brane

probes can be found in 22]. Of course one may wish first to generalize the above collinear non-extremal charged black hole solutions to arbitrary space-time dimensions. 


\section{Acknowledgments}

We would like to thank Frank Ferrari and Lori Paniak for many discussions. MSC is

supported by FCT (Portugal) under programme PRAXIS XXI and by the NSF grant PHY-9802484.

\section{References}

[1] W. Israel and K.A. Khan, Nuovo Cimento 33 (1964) 331.

[2] H. Weyl, Ann. Physik 54 (1917) 117; Ann. Physik 54 (1917) 185.

[3] A. Einstein and N. Rosen, Phys. Rev. 49 (1936) 404.

[4] H. Muller zum Hagen and H.J. Seifert, Int. J. Theor. Phys. 8 (1973) 443.

[5] G.W. Gibbons, Commun. Math. Phys. 35 (1974) 13.

[6] G.W. Gibbons and M.J. Perry, Phys. Rev. D22 (1980) 313.

[7] T. Regge, Nuovo Cimento 19 (1961) 558.

[8] I.R. Klebanov and A.A. Tseytlin, Nucl. Phys. B475 (1996) 179, hep-th/9604166.

[9] M. Cvetic and A.A. Tseytlin, Nucl. Phys. B478 (1996) 181, hep-th/9606033.

[10] J. Maldacena and A. Strominger, Phys. Rev. D55 (1997) 861, hep-th/9609026.

[11] J.M. Maldacena, Adv. Theor. Math. Phys. 2 (1998) 231, hep-th/9711200.

[12] S.S. Gubser, I.R. Klebanov and A.M. Polyakov, Phys. Lett. B428 (1998) 105, hepth/9802109.

[13] E. Witten, Adv. Theor. Math. Phys. 2 (1998) 253, hep-th/9802150.

[14] A. Strominger and C. Vafa, Phys. Lett. B379 (1996) 99, hep-th/9601029.

[15] J.M. Maldacena, Nucl. Phys. B477 (1996) 168, hep-th/9605016.

[16] S.R. Das and S.D. Mathur, Nucl. Phys. B478 (1996) 561, hep-th/9606185; Nucl. Phys. B482 (1996) 153, hep-th/9607149. 
[17] C.G. Callan, S.S. Gubser, I.R. Klebanov and A.A. Tseytlin, Nucl. Phys. B489 (1997) 65, hep-th/9610172.

[18] S.F. Hassan and Spenta R. Wadia, Phys. Lett. B402 (1997) 43, hep-th/9703163; Nucl. Phys. B526 (1998) 311, hep-th/9712213.

[19] M.S. Costa and M.J. Perry, Nucl. Phys. B524 (1998) 333, hep-th/9712160; M.S. Costa, JHEP 11 (1998) 007, hep-th/9807185.

[20] R.C. Rashkov and K.S. Viswanathan, Phys. Rev. D62 (2000) 046009, hep-th/9911160.

[21] M.S. Costa, JHEP 05 (2000) 041, hep-th/9912073; Phys. Lett. B482 (2000) 287, hep-th/0003289;

[22] E. Kiritsis, JHEP 10 (1999) 010, hep-th/9906206. 